\title{
Comparison and characterization of $\alpha$-amylase inducers in Aspergillus nidulans based on nuclear localization of AmyR
}

\author{
Yuriko Murakoshi • Tomohiro Makita • Masashi Kato • \\ Tetsuo Kobayashi
}

Received: 20 November 2011 / Revised: 19 December 2011 / Accepted: 26 December 2011 /Published online: 18 January 2012

(C) The Author(s) 2012. This article is published with open access at Springerlink.com

\begin{abstract}
AmyR, a fungal transcriptional activator responsible for induction of amylolytic genes in Aspergillus nidulans, localizes to the nucleus in response to the physiological inducer isomaltose. Maltose, kojibiose, and D-glucose were also found to trigger the nuclear localization of GFP-AmyR. Isomaltose- and kojibiose-triggered nuclear localization was not inhibited by the glucosidase inhibitor, castanospermine, while maltose-triggered localization was inhibited. Thus, maltose itself does not appear to be an direct inducer, but its degraded or transglycosylated product does. Non-metabolizable D-glucose analogues were also able to trigger the nuclear localization, implying that these sugars, except maltose, directly function as the inducers of AmyR nuclear entry. The inducing activity of D-glucose was 4 orders-of-magnitude weaker compared with isomaltose. Although D-glucose has the ability to induce $\alpha$-amylase production, this activity would generally be masked by CreA-dependent carbon catabolite repression. Significant induction of $\alpha$-amylase by D-glucose was observed in creA-defective A. nidulans.
\end{abstract}

Keywords Aspergillus nidulans · Amylase · Inducer · AmyR · Nuclear localization

Y. Murakoshi · T. Makita $\cdot$ M. Kato $\cdot$ T. Kobayashi $(\bowtie)$

Department of Biological Mechanisms and Functions, Graduate School of Bioagricultural Sciences, Nagoya University, Furo-cho, Chikusa-ku,

Nagoya-shi, Aichi 464-8601, Japan

e-mail:koba@agr.nagoya-u.ac.jp

\section{Introduction}

Filamentous fungi are widely used for industrial production of biomass-degrading enzymes including amylases, xylanases, cellulases, and lipases. These enzymes are generally produced as a mixture of enzymes with a similar function, such as amylolytic enzymes, upon induction by a specific inducing molecule so called as an inducer. Maximal production of the enzymes may be achieved by appropriate control of the inducer concentration. However, it seems that not much attention has been paid in this context, considering that a real inducer, or a physiological inducer in other words, remains to be identified even for production of amylases, the most abundantly produced enzymes in industries.

Amylolytic enzymes, such as $\alpha$-amylase, glucoamylase, and $\alpha$-glucosidase, are inducible enzymes in Aspergillus species. Previous studies have shown that $\alpha$-amylase production in Aspergillus oryzae and A. nidulans is induced by various $\alpha$-linked glucobioses and glucotrioses including kojibiose, maltose, isomaltose, and panose (Tonomura et al. 1961; Kato et al. 2002a). Isomaltose was proposed to be the physiological inducer in $A$. nidulans, since it has the strongest inducing activity examined to date. It exists in culture supernatants when the organism is grown on either maltose or starch, and does not require enzymatic conversion to exhibit inducing activity (Kato et al. 2002a).

Glucose is generally recognized as a repressing carbon source of amylase production in Aspergillus spp. However, it has been reported in A. oryzae and A. niger that, in addition to acting as a repressing carbon source because of the CreA protein, D-glucose also acts as an inducer of $\alpha$-amylase 
production (Carlsen and Nielsen 2001; Vankuyk et al. 2012). These findings raise the possibility that D-glucose by itself, without any enzymatic conversions, may induce expression of the amylolytic genes.

AmyR is the transcriptional activator responsible for induction of the amylolytic genes in Aspergillus species (Petersen et al. 1999; Gomi et al. 2000; Tani et al. 2001b; Nakamura et al. 2006). It has a $\mathrm{Zn}(\mathrm{II})_{2} \mathrm{Cys}_{6}$ DNA binding motif at its $\mathrm{N}$ terminus and binds to the $\mathrm{CGGN}_{8}(\mathrm{C} / \mathrm{A}) \mathrm{GG}$ sequence present in the amylase promoters (Tani et al. 2001a; Ito et al. 2004). Transcription of the amylase genes is controlled by regulated nuclear localization of AmyR; the physiological inducer isomaltose triggers nuclear accumulation of AmyR (Makita et al. 2009). In this report, we compared the ability of various sugars to trigger the nuclear localization of AmyR.

\section{Materials and methods}

Strains and growth conditions

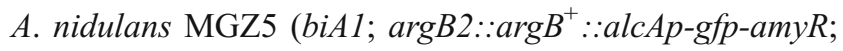
pyroA4; amyR1/malA1) carrying the GFP-AmyR fusion gene under control of the alcA promoter (Makita et al. 2009) was used for sub-cellular localization studies. The strain ABTA16 (biA1 pyrG89; wA3; $\operatorname{argB2}:: \arg B^{+}:: t a a G 2$; pyroA4) carries a single copy of the $A$. oryzae taa $G 2$ gene in the $\arg B$ locus (Kato et al. 2002a). The strain CT3 (biA1

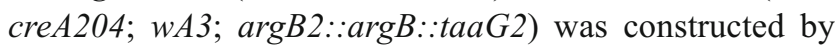
crossing the strains ABTA16 and CA4 (creA204 biAl; wA3; $\arg B 2$ ), the latter strain being progeny of a cross between ABPU1 (biA1 pyrG89; wA3; $\operatorname{argB2}$; pyroA4) and MH664 (FGSC A748) (creA204 biA1; niiA4). A. nidulans strains were grown at $37^{\circ} \mathrm{C}$ in the standard minimal medium (Rowlands and Turner 1973) supplemented with appropriate requirements, unless otherwise stated.

\section{Fluorescence microscopy}

Fluorescence microscopy was performed as described previously (Makita et al. 2009). Briefly, A. nidulans spores were inoculated on cover slips dipped in minimal medium with $2 \%$ lactose as the sole carbon source and incubated at $37{ }^{\circ} \mathrm{C}$ for $14 \mathrm{~h}$. Ethyl methyl ketone was then added at a concentration of $50 \mathrm{mM}$ to trigger the alcA promoterdependent expression of the GFP-AmyR gene. After incubation at $37^{\circ} \mathrm{C}$ for $3 \mathrm{~h}$, amylase inducers were added at various concentrations and incubated at $37{ }^{\circ} \mathrm{C}$ for $15 \mathrm{~min}$ or $60 \mathrm{~min}$. The mycelia on the cover slips were examined by fluorescent microscopy. All microscopy and image preparations were performed with an Olympus BX51TRF microscope equipped with a $40 \times$ UPlanApo objective lens set. Olympus filter units, U-MGFPHQ and U-MWU, were used for detection of GFP and DAPI fluorescence, respectively. Images were captured with a cool CCD camera (Hamamatsu Photonics) controlled by the AQUA-LITE software. Experiments were repeated at least three times for each amylase inducers under the identical conditions.

$\alpha$-Amylase assay

The $\alpha$-amylase assay was performed as described previously (Kato et al. 2002a). A. nidulans strains were pre-grown for $24 \mathrm{~h}$ in the minimal medium with D-glucose replaced by $2 \%$ polypeptone (Wako Chemicals Ltd., Japan). After washing the mycelia with the standard minimal medium lacking carbon sources, the mycelia was suspended in a fresh medium of the same composition as used for the pre-culture. Amylase inducers were then added. $\alpha$-Amylase activity in the culture filtrates was measured after $12 \mathrm{~h}$ cultivation as previously described (Kato et al. 2002a).

Chemicals

Maltose and isomaltose were purchased from Sigma (St. Louis, MO, USA) and Seikagakukogyo (Tokyo, Japan), respectively. Kojibiose was kindly provided by Kikkoman Co., Ltd. (Chiba, Japan). Castanospermine was obtained from BIOMOL Research Laboratories, Inc. (Plymouth Meeting, PA, USA). All other chemicals were of reagent grade.

\section{Results}

Amylase inducers trigger nuclear localization of AmyR

$\alpha$-Glucobioses, such as kojibiose (2-O- $\alpha$-glucosylglucose), maltose (4-O- $\alpha$-glucosylglucose), and isomaltose (6- $O-\alpha$-glucosylglucose), induce amylase synthesis in $A$. nidulans (Kato et al. 2002a). Isomaltose is the strongest inducer, inducing amylase synthesis at $3 \mu \mathrm{M}$; kojibiose and maltose are moderate inducers, exhibiting inducing activity at $0.3 \mathrm{mM}$ and above.

We have previously shown that isomaltose triggers rapid nuclear accumulation of GFP-AmyR (Makita et al. 2009). The ability of kojibiose and maltose to trigger the nuclear accumulation of GFP-AmyR was examined to confirm that AmyR localizes to the nuclei in the presence of other amylase inducers (Fig. 1). In the absence of the inducers, GFP fluorescence was distributed in the cytosol of the hyphae. Fifteen minutes after the addition of isomaltose and kojibiose, GFP fluorescence accumulated in the nuclei (bright small dots in Fig. 1). Maltose-triggered nuclear accumulation was not observed at $15 \mathrm{~min}$, but was present at $60 \mathrm{~min}$.

When an $\alpha$ - and $\beta$-glucosidase inhibitor, castanospermine, was added, maltose-triggered nuclear localization was inhibited. 
Fig. 1 Inducer-dependent nuclear localization of GFPAmyR. Isomaltose, kojibiose, and maltose were added at a concentration of $3 \mathrm{mM}$. $+\mathrm{CS}$ indicates that castanospermine was added at a concentration of $0.3 \mathrm{mM}$ prior to the addition of the inducers. The samples were examined at 15 and $60 \mathrm{~min}$ after the addition of the inducers

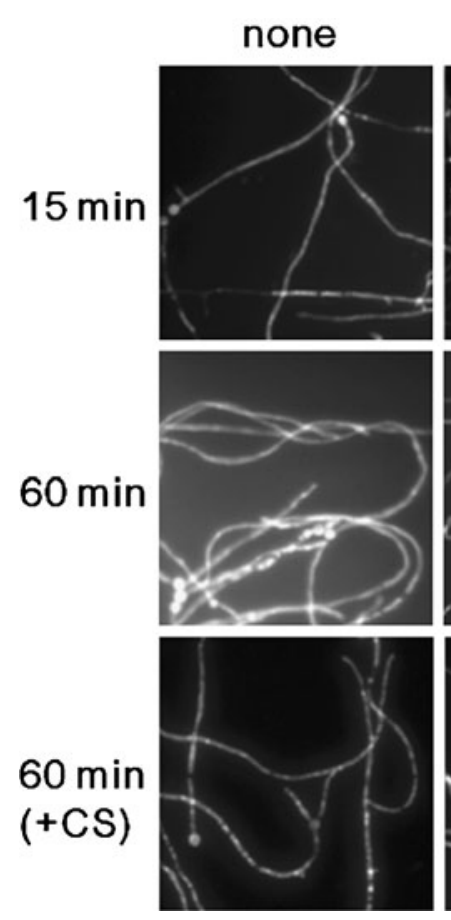

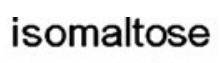
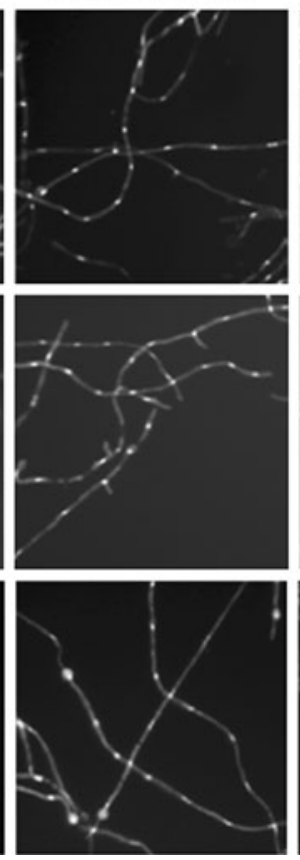

kojibiose
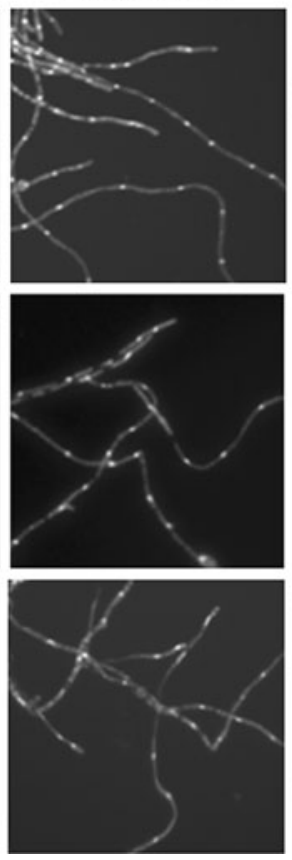

maltose
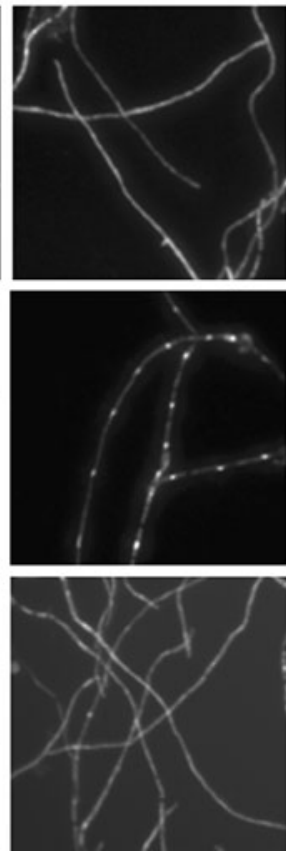

In contrast, isomaltose and kojibiose were still able to trigger the accumulation (Fig. 1). As previously reported, castanospermine inhibits degradation of isomaltose in the culture (Kato et al. 2002a); this is also the case for kojibiose (data not shown). These results indicate that maltose requires a pre-existing $\alpha$ - or $\beta$-glucosidase(s) to trigger the nuclear localization of AmyR, while isomaltose and kojibiose do not. This is consistent with our previous findings that maltose induction of $\alpha$-amylase requires a pre-existing $\alpha$-glucosidase(s) with isomaltoseproducing activity and that isomaltose and kojibiose do not require such an enzyme for this induction (Kato et al. 2002a, b). Based on these findings, we propose that maltose itself is not a direct inducer of $\alpha$-amylase production at least in A. nidulans.

Glucose, a repressing carbon source, triggers nuclear localization

Although D-glucose generally represses the expression of amylolytic genes in a CreA-dependent manner (Felenbok and Kelly 1996), it has also been shown to induce $\alpha$ amylase in A. oryzae (Carlsen and Nielsen 2001). In order to further explore the effects of D-glucose on $\alpha$-amylase production, sub-cellular localization of GFP-AmyR was examined after $15 \mathrm{~min}$ of D-glucose addition and at the final concentration of $3 \mathrm{mM}$. As shown in Fig. 2a and b, Dglucose triggered nuclear localization of GFP-AmyR, but D-galactose and D-xylose did not. D-Mannose very weakly triggered the nuclear localization; strong GFP fluorescence was observed only in some nuclei at the concentration of $3 \mathrm{mM}$. While D-glucose induced amylase activity in a creA deficient strain as shown below, D-mannose did not (data not shown), suggesting that D-mannose-triggered nuclear accumulation of AmyR is not sufficient to produce detectable amounts of $\alpha$-amylase. These results indicate that only D-glucose, among the monosaccharides examined, had the ability to trigger substantial nuclear accumulation of AmyR. In addition, rapid nuclear accumulation (within $15 \mathrm{~min}$ ) in response to D-glucose suggests that D-glucose directly, without any enzymatic conversion, triggers this nuclear accumulation in the same manner as isomaltose and kojibiose.

To confirm this, non-metabolizable D-glucose analogues, 1-deoxyglucose, 2-deoxyglucose, and 6-deoxyglucose, were examined for the ability to trigger the nuclear accumulation. GFP-AmyR accumulated in the nucleus within $15 \mathrm{~min}$ of their addition (Fig. 2c). Consequently, D-glucose, without any enzymatic conversion, apparently functions as an inducer of this nuclear accumulation. In addition, these results imply that the hydroxyl groups at $\mathrm{C} 1, \mathrm{C} 2$, and $\mathrm{C} 6$ of D-glucose are not essential.

Comparison of effective concentrations of isomaltose, kojibiose, and D-glucose required for triggering the nuclear accumulation revealed the exceptional activity of isomaltose. GFP-AmyR clearly accumulated in the nuclei at $0.3 \mu \mathrm{M}$ of isomaltose (Fig. 3). In comparison, $30 \mu \mathrm{M}$ and $3 \mathrm{mM}$ concentrations were required for kojibiose and D-glucose, respectively, to demonstrate an equivalent nuclear accumulation. Thus, kojibiose and D-glucose appeared to be approximately 
Fig. 2 Ability of various monosaccharides to trigger the nuclear localization of GFP-AmyR. All monosaccharides were added at a concentration of $3 \mathrm{mM}$, and microscopic observation was performed at $15 \mathrm{~min}$ after their addition. a D-Glucose was added and the same mycelia were observed by DAPI (left) and GFP (right) fluorescence. b GFP fluorescence after addition of D-glucose, D-mannose, D-galactose, and D-xylose. c GFP fluorescence after addition of 1-deoxyglucose (1-DOG), 2-deoxyglucose $(2-D O G)$, and 6-deoxyglucose (6-DOG), whose structures are shown
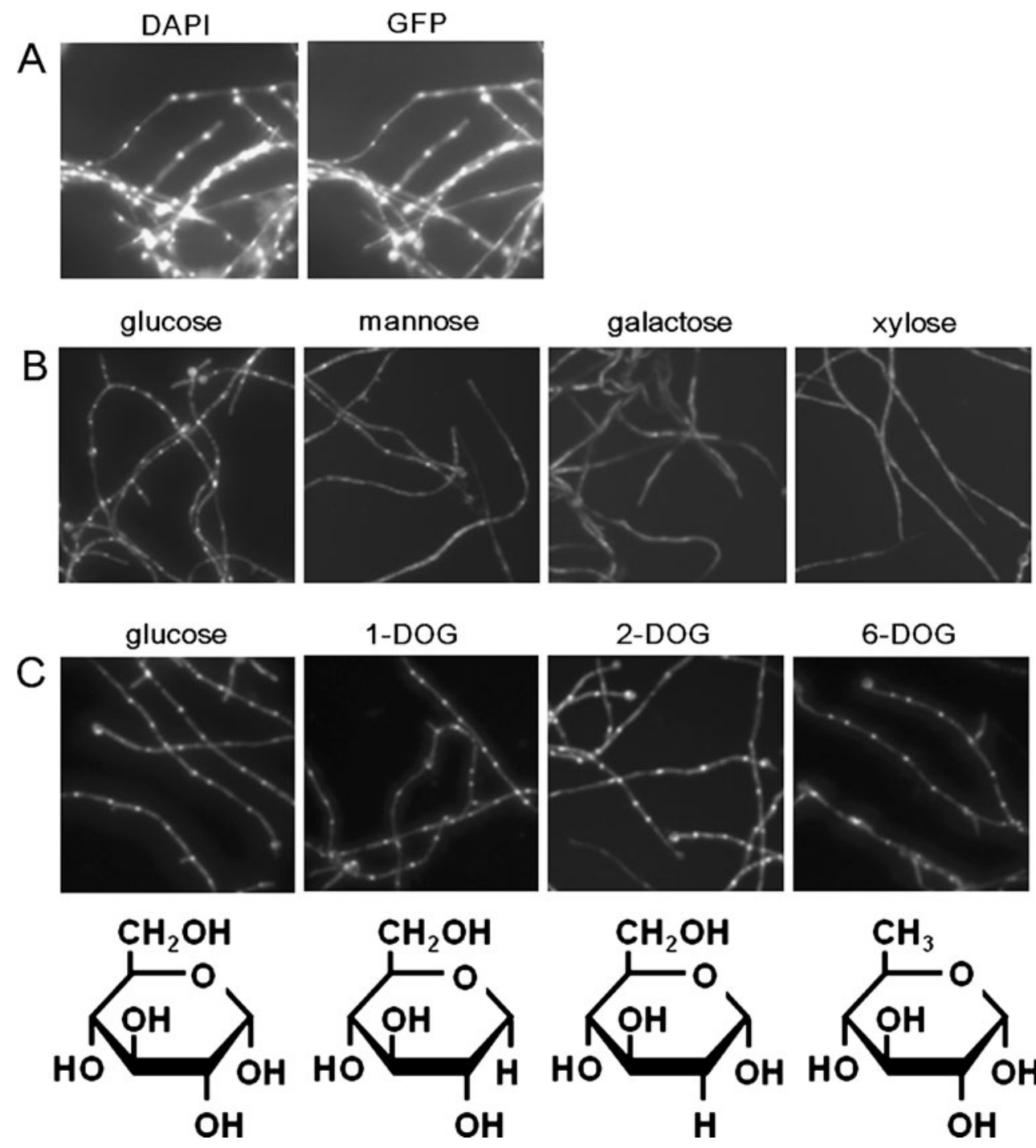

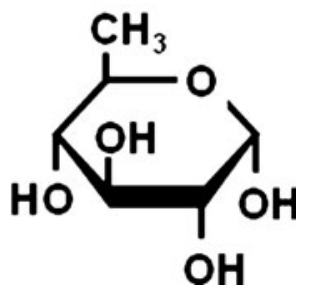

2 and 4 orders-of-magnitude weaker compared with isomaltose when added into the culture.

Glucose induces $\alpha$-amylase production in a CreA-deficient strain of $A$. nidulans

To examine whether D-glucose induces $\alpha$-amylase production, we used $A$. nidulans strains that carry the Taka-amylase A gene (taaG2) of $A$. oryzae. The taaG2 gene is inducibly and abundantly expressed in A. nidulans and has been used as a reporter gene for examination of amylase gene regulation in this species (Tani et al. 2001a, b; Ito et al. 2004; Makita et al. 2009).

The strain ABTA16 $\left(\mathrm{creA}^{+}\right.$, amy $\left.R^{+}\right)$produced approximately 60 times higher $\alpha$-amylase activity in the presence of $0.1 \%$ isomaltose compared with its absence (Fig. 4). When $0.1 \%$ Dglucose was used, $\alpha$-amylase production was decreased approximately eight times compared with that observed in the presence of isomaltose. In this instance, the observed activity was seven times higher compared with that seen in the non-induced condition. At the higher D-glucose concentration of $2 \%, \alpha$-amylase production decreased to half of that seen with $0.1 \%$ D-glucose. $\alpha$-Amylase induction by D-glucose, and isomaltose, was not detected in the strain $\Delta$ amyR103 (cre $\left.A^{+}, \Delta a m y R\right)$ (Tani et al. 2001b) (data not shown). This indicates that D-glucose induces $\alpha$-amylase production in an AmyR-dependent manner, as expected from the results of the sub-cellular localization studies described above.

In the CreA mutant, CT3 (creA204, amy $\left.R^{+}\right), \alpha$-amylase activity was higher at $2 \% \mathrm{D}$-glucose compared with $0.1 \%$. In addition, 2-deoxyglucose at $0.1 \%$ induced comparable $\alpha$ amylase activity to that observed with $2 \%$ D-glucose. These results clearly indicate that D-glucose induces production of $\alpha$-amylase, although it represses this production in a CreAdependent manner. 
Fig. 3 Comparison of activity of isomaltose, kojibiose, and glucose to trigger the nuclear localization of GFP-AmyR. The sugars were added at the indicated concentrations and the GFP fluorescence was examined at $15 \mathrm{~min}$ after their addition
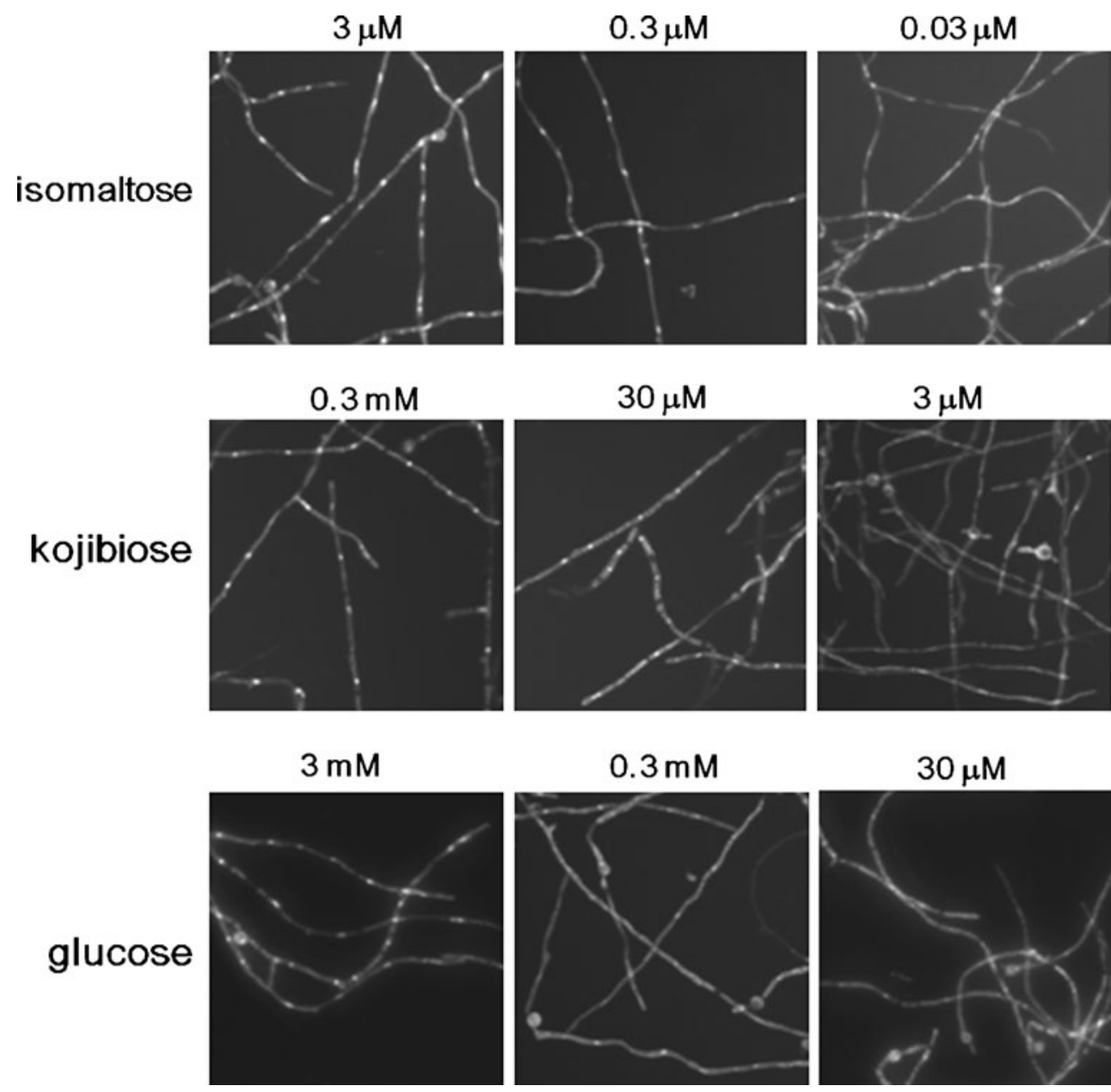

\section{Discussion}

Various $\alpha$-linked glucobioses induce amylase activity in $A$. oryzae and $A$. nidulans, strongest inducer being isomaltose (Tonomura et al. 1961; Kato et al. 2002a). It also appeared to be an extremely effective molecule to cause nuclear localization of AmyR, triggering this within a short period and at very low concentrations (Figs. 1 and 3). Maltosetriggered nuclear localization of AmyR required glucosidase activity, but isomaltose and kojibiose did not. These observations concerning the ability of the glucobioses in triggering the nuclear localization are analogous to those in amylase induction (Kato et al. 2002a), indicating that the activity to trigger the nuclear localization probably determines the strength of the amylase inducers.

Besides acting as a repressing carbon source, D-glucose acts as an amylase inducer in A. oryzae (Carlsen and Nielsen 2001). Previous studies had failed to clarify whether the Dglucose directly, without any enzymatic conversions, activates AmyR-mediated expression of the amylolytic genes. Our results, from the studies using non-metabolizable D-glucose analogues, suggest that D-glucose directly triggers nuclear localization of AmyR (Fig. 3). The activity of D-glucose to trigger nuclear localization is much weaker compared with isomaltose and kojibiose. This weak activity, together with its

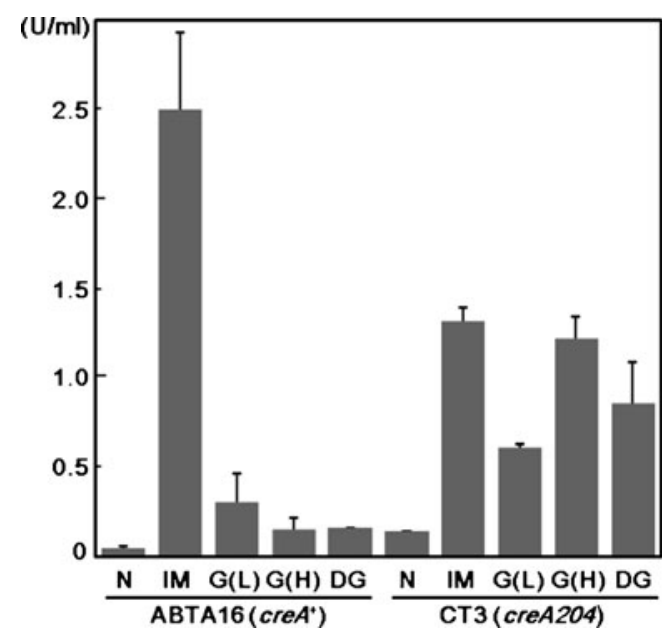

Fig. $4 \alpha$-Amylase induction in A. nidulans cre $^{+}$and creA204 strains. Isomaltose $(I M)$ and 2-deoxyglucose $(D G)$ were added at a concentration of $0.1 \%$. The concentrations of D-glucose were $0.1 \%$ and $2 \%$, which are indicated as $\mathrm{G}(\mathrm{L})$ and $\mathrm{G}(\mathrm{H})$, respectively. $N$ denotes no inducing carbon sources such as isomaltose, 2-deoxyglucose, and D-glucose 
nature to cause strong carbon catabolite repression, meant that sufficient amylase induction by D-glucose could be detected only under a CreA-defective background (Fig. 4).

When starch is present as the sole carbon source, some portion of starch must be first degraded, prior to amylase induction, by $\alpha$-amylases, glucoamylases and $\alpha$-glucosidases produced at the basal level, forming maltooligosaccharides, maltose, and D-glucose. At the same time, strong $\alpha-1,6$-bondforming transglycosylation activity of some $\alpha$-glucosidases such as AgdA and AgdB would lead to formation of isomaltose, the strongest inducer reported in A. nidulans (Kato et al. 2002a, b). Consequently, two direct inducers, D-glucose and isomaltose, are expected to be present in the starch-grown culture, and this is also the case when maltose is used as the sole carbon source. Approximately the same amounts of Dglucose and isomaltose were detected in the culture broth at $4 \mathrm{~h}$ after the induction with $0.1 \%$ maltose (data not shown). Considering that D-glucose is a much weaker inducer compared with isomaltose in triggering the nuclear localization of AmyR, and that high levels of D-glucose causes CreA-dependent repression, D-glucose would be expected to contribute little in the induction of $\alpha$-amylase when grown on maltose or starch. Thus, in laboratory conditions using pure culture, high level of $\alpha$-amylase production with D-glucose could be achieved only when it is supplied continuously at low concentrations.

However, this situation may be altered in conditions where both starch and high concentrations of D-glucose are present. While CreA represses transcription of amylolytic genes directly and indirectly because of repression of the $a m y R$ expression (Tani et al. 2001b), AmyR existing at the basal level does accumulate in the nuclei, ready to transactivate its targets. Decrease in the environmental concentration of D-glucose would immediately lead to transcription of amylolytic genes and $a m y R$ itself, which would be followed by a boost in production of amylolytic enzymes through production of the strongest inducer, isomaltose.

The reason why D-glucose is not the most effective inducer might be speculated from the fact that the major sources of D-glucose in nature are starch and cellulose. If AmyR responded to very low concentrations of D-glucose, high levels of $\alpha$-amylase would be induced by cellulose. It should also be noted that currently known cellulase inducers are not D-glucose but sophorose in Trichoderma viride (Mandels et al. 1962), gentiobiose in Penicillium purpurogenum (Kurasawa et al. 1992), and D-xylose in A. niger and A. oryzae (van Peij et al. 1998; Marui et al. 2002). The glucobioses sophorose and gentiobiose are shown to be produced by the action of $\beta$-glucosidase (Kurasawa et al. 1992; Mach et al. 1995), which is analogous to isomaltose formation by $\alpha$-glucosidases (Kato et al. 2002a, b).

In our previous report, expression of amyR was shown to be repressed in a CreA-dependent manner (Tani et al. 2001b). Expression of amy $R$ was highest on starch followed by maltose, and decreased on D-glucose and glycerol in the $\mathrm{creA}^{+}$strain of A. nidulans. In the creA204 strain, the expression levels were almost identical on starch, maltose, and D-glucose. The expression level was still low on glycerol, but we could not find a reasonable explanation for this finding. The current results, showing that D-glucose is one of the inducers, suggest the explanation may be that expression of the $a m y R$ gene is under control of AmyR itself. Further evidence to support this is the presence of an AmyR binding site, $\mathrm{CGGN}_{8} \mathrm{CGG}$, at -232 to -245 (referring the translational start site as +1 ) in the promoter region of the $a m y R$ gene.

Acknowledgement This work was partially supported by a Grant-inAid for Scientific Research (B) and (C) to T. K. from the Ministry of Education, Science, Sports and Culture.

Open Access This article is distributed under the terms of the Creative Commons Attribution Noncommercial License which permits any noncommercial use, distribution, and reproduction in any medium, provided the original author(s) and source are credited.

\section{References}

Carlsen M, Nielsen J (2001) Influence of carbon source on $\alpha$-amylase production by Aspergillus oryzae. Appl Microbiol Biotechnol 57:346-349

Felenbok B, Kelly JM (1996) Regulation of carbon metabolism in mycelial fungi. In The Mycota III. Biochemistry and Molecular Biology. Brambl and Marzluf. Springer-Verlag, Berlin, pp 369380

Gomi K, Akeno T, Minetoki T, Ozeki K, Kumagai C, Iimura Y (2000) Molecular cloning and characterization of a transcriptional activator gene, amy $R$, involved in the amylolytic gene expresssion in Aspergillus oryzae. Biosci Biotechnol Biochem 64:816-827

Ito T, Tani S, Itoh T, Tsukagoshi N, Kato M, Kobayashi T (2004) Mode of AmyR binding to the $\mathrm{CGGN}_{8} \mathrm{AGG}$ sequence in the Aspergillus oryzae taaG2 promoter. Biosci Biotechnol Biochem 68:1906-1911

Kato N, Murakoshi Y, Kato M, Kobayashi T, Tsukagoshi N (2002a) Isomaltose formed by $\alpha$-glucosidases triggers amylase induction in Aspergillus nidulans. Curr Genet 42:43-50

Kato N, Suyama S, Shirokane M, Kato M, Kobayashi T, Tsukagoshi N (2002b) Novel $\alpha$-glucosidase from Aspergillus nidulans with strong transglycosylation activity. Appl Environ Microbiol 68:1250-1256

Kurasawa T, Yachi M, Suto M, Kamagata Y, Takao S, Tomita F (1992) Induction of cellulase by gentiobiose and its sulfur-containing analog in Penicillium purpurogenum. Appl Environ Microbiol 58:106-110

Mach RL, Seiboth B, Myasnikov A, Gonzalez R, Strauss J, Harkki AM, Kubicek CP (1995) The bgll gene of Trichoderma reesei QM9414 encodes an extracellular, cellulose-inducible $\beta$-glucosidase involved in cellulase induction by sophorose. Mol Microbiol 16:687-697

Makita T, Katsuyama Y, Tani S, Suzuki H, Kato N, Todd RB, Hynes MJ, Tsukagoshi N, Kato M, Kobayashi T (2009) Inducerdependent nuclear localization of a $\mathrm{Zn}(\mathrm{II})_{2} \mathrm{Cys}_{6}$ transcriptional activator, AmyR, in Aspergillus nidulans. Biosci Biotechnol Biochem 73:391-399

Mandels M, Parrish FW, Reese ET (1962) Sophorose as an inducer of cellulase in Trichoderma viridae. J Bacteriol 83:400-408

Marui J, Kitamoto N, Kato M, Kobayashi T, Tsukagoshi N (2002) Transcriptional activator, AoXlnR, mediates cellulose-inductive 
expression of the xylanolytic and cellulolytic genes in Aspergillus oryzae. FEBS Lett 528:279-282

Nakamura T, Makita T, Maeda Y, Tanoue N, Kato M, Kobayashi T (2006) Expression profile of amylolytic genes in Aspergillus nidulans. Biosci Biotechnol Biochem 70:2363-2370

Petersen KL, Lehmbeck J, Christensen T (1999) A new transcriptional activator for amylase genes in Aspergillus. Mol Gen Genet 262:668-676

Rowlands RT, Turner G (1973) Nuclear and extranuclear inheritance of oligomycin resistance in Aspergillus nidulans. Mol Gen Genet 126:201-216

Tani S, Itoh T, Kato M, Kobayashi T, Tsukagoshi N (2001a) In vivo and in vitro analyses of the AmyR binding site of the Aspergillus nidulans agdA promoter; requirement of the CGG direct repeat for induction and high affinity binding of AmyR. Biosci Biotechnol Biochem 65:1568-1574
Tani S, Katsuyama Y, Hayashi T, Suzuki H, Kato M, Gomi K, Kobayashi T, Tsukagoshi N (2001b) Characterization of the $a m y R$ gene encoding a transcriptional activator for the amylase genes in Aspergillus nidulans. Curr Genet 39:10 15

Tonomura K, Suzuki H, Nakamura N, Kuraya K, Tanabe O (1961) On the inducers of $\alpha$-amylase formation in Aspergillus oryzae. Agric Biol Chem 25:1-6

van Peij NN, Gielkens MM, de Vries RP, Visser J, de Graaff LH (1998) The transcriptional activator XlnR regulates both xylanolytic and endoglucanase gene expression in Aspergillus niger. Appl Environ Microbiol 64:3615-3619

Vankuyk PA, Benen JA, Wosten HA, Visser J, de Vries RP (2012) A broader role for AmyR in Aspergillus niger: regulation of the utilisation of D-glucose or D-galactose containing oligo- and polysaccharides. Appl Microbiol Biotechnol 93:258-293 